\title{
Eliciting Gul's theory of disappointment aversion by the tradeoff method
}

\author{
Mohammed Abdellaoui ${ }^{a}$, Han Bleichrodt ${ }^{b, *}$ \\ ${ }^{a}$ CNRS-GREGHEC \& HEC School of Management, Paris 1, Rue de la Libération F-78351 Jouy-en-Josas, France \\ ${ }^{\mathrm{b}}$ Department of Economics, H13-27, Erasmus University, P.O. Box 1738, 3000 DR Rotterdam, The Netherlands
}

Received 15 June 2006; received in revised form 28 March 2007; accepted 26 July 2007

Available online 1 September 2007

\begin{abstract}
Gul's theory of disappointment aversion (DA) has several attractive features, being intuitive, analytically tractable, and parsimonious. In spite of this, the DA model has received little attention in practical applications, which may be partly due to the absence of a procedure to elicit the model. We show how the tradeoff method, developed by Wakker and Deneffe can be used to elicit DA. Our elicitation method is parameter-free: it requires no assumption about utility and/or disappointment aversion. Quantitative tests of DA in three outcome domains, monetary gains, monetary losses, and life-years, suggest that the DA model is too parsimonious. Of the other models of disappointment aversion that have been proposed in the literature, our data are most consistent with the model of Loomes and Sugden.

(c) 2007 Elsevier B.V. All rights reserved.
\end{abstract}

JEL classification: D81

PsycINFO classification: $2200 ; 2340$

Keywords: Disappointment aversion; Tradeoff method; Nonexpected utility; Probability weighting; Sign-dependence

\footnotetext{
* Corresponding author. Tel.: +31 104081295 .

E-mail addresses: abdellaoui@hec.fr (M. Abdellaoui), bleichrodt@few.eur.nl (H. Bleichrodt).
} 


\section{Introduction}

In response to the observed violations of expected utility, several generalizations of expected utility have been proposed (Starmer, 2000). Among these generalizations, Gul's (1991) theory of Disappointment Aversion (DA) has several attractive features. First it is intuitive, being based on the idea that a decision maker when evaluating a prospect forms a prior expectation and experiences disappointment when the prospect results in an outcome that is worse than this prior expectation. Second, DA is analytically tractable. Third, DA is a parsimonious theory, being only one parameter richer than expected utility. This extra parameter reflects people's disappointment aversion. DA's parsimony is an important advantage in applications. A formal definition of the DA model, which explains the model's characteristics in detail, is given in Section 2.

Several other disappointment models exist which are based on a similar intuition as DA (Bell, 1985; Delquié \& Cillo, 2006a, 2006b; Grant \& Kajii, 1998; Jia, Dyer, \& Butler, 2001; Loomes \& Sugden, 1986). The class of preferences considered in these models is different from DA, however. The main difference is that in DA the decision maker's prior expectation of a prospect is endogenously determined, whereas in the other disappointment models the prior expectation is exogenously determined. We will briefly consider these alternative models in Section 6.

In spite of its desirable features, DA has not been used in applications. One reason for this neglect might be that no straightforward procedure exists to elicit the model. Traditional utility measurement techniques, such as probability equivalence, certainty equivalence, and lottery equivalence (Farquhar, 1984; McCord \& de Neufville, 1986), cannot be used because they are vulnerable to violations of expected utility. Wakker and Deneffe (1996) developed the tradeoff method to measure utility when people behave according to rank-dependent utility (Quiggin, 1981; Yaari, 1987), i.e., when they weight probabilities. We show that in the choices involved in the tradeoff method, DA is a special case of rank-dependent utility and, hence, the tradeoff method can also be used to elicit utility under DA. Once utility is known, the elicitation of the disappointment aversion parameter is straightforward. The tradeoff method allows to elicit the complete DA model and requires no assumptions about utility or disappointment aversion.

The availability of a procedure to elicit DA makes it possible to test the restrictions imposed by DA. We used data from Abdellaoui (2000) and Bleichrodt and Pinto (2000) to test DA. Taken together, these data allow a test of DA in three outcome domains, monetary gains, monetary losses, and life-years, using both hypothetical and incentivized questions. A priori there is some reason to doubt the descriptive validity of DA. As we show in Section 2, as a special case of rank-dependent utility, DA does not allow for the most commonly observed pattern of probability weighting. The test of DA is interesting nevertheless as the deviations from DA can suggest ways to extend the model.

Previous evidence on the DA model yielded mixed results. Camerer and Ho (1994) tested betweenness, a central assumption of DA, and found systematic violations. Loomes and Segal (1994) tested the order of magnitude of decision makers' attitudes towards risk and found no support for the pattern predicted by DA. Hey and Orme (1994) found that DA did not fit their data well. On the other hand, when Camerer and Ho (1994) allowed for random error and fitted stochastic choice models to their data, DA accommodated the data well and provided a comparable fit as prospect theory, the leading descriptive theory of decision under risk. Morone and Schmidt (2002) found that, among expected utility and 
several important generalizations of expected utility, DA most accurately predicted willingness to accept and willingness to pay responses. Blavatskyy (2006) showed that most previously observed violations of betweenness could be explained by random errors. Harless and Camerer (1994) concluded that there is room for a theory that is richer than EU in the sense of being able to explain a few common violations of expected utility, but that is leaner than many of the generalizations of expected utility that allow a lot of patterns that are rarely observed. This description might fit DA. As noted above, the DA model is only one parameter richer than expected utility and the deviations from expected utility that it allows are commonly observed. For example, in the unit triangle DA allows for the commonly observed mixed fanning pattern, fanning in for relatively attractive prospects, i.e., prospects in the northwest corner of the triangle, and fanning out for relatively unattractive prospects, prospects in the southeast corner of the triangle (Chew \& Waller, 1986; Conlisk, 1989; Neilson, 1992; Starmer, 2000).

A quantitative measurement of disappointment aversion is not only important for descriptive purposes, but also for applied decision analysis, where prescriptions have to be made. There is no consensus on the prescriptive status of disappointment models. Loomes and Sugden (1986) defend the normative status of disappointment. They argue that when people consistently maximize expected satisfaction where that expectation includes the anticipation of possible disappointment and elation, there is no reason why such a maximand would be irrational. Similar arguments have been put forward by Bell (1985) and Delquié and Cillo (2006a). Others, who have recognized the descriptive limitations of expected utility have been more reluctant to abandon the normative validity of expected utility (e.g. Kahneman \& Tversky, 1979; Savage, 1954). Irrespective of the prescriptive status of the DA model, it is important for applied decision analysis to be able to measure disappointment if disappointment affects decision makers' preferences. This is clearly the case if the DA model is considered normative because then feelings of disappointment should be included in the analysis. However, it is also true if the DA model is merely considered descriptive. Prescriptive decision making require the measurement of utilities. Measuring utilities is a descriptive task and, hence, susceptible to the biases that lead to violations of expected utility. To correct for these biases a method is needed that can separate utility from other factors affecting preferences. Our measurement method allows such a separation between disappointment and utility. Hence, if disappointment affects a decision maker's preferences and expected utility is considered normative then our method can be used to correct expected utilities for disappointment. As we explain in Section 3, our method can also be useful in the correction of utility measurements from traditional methods such as the probability equivalence and certainty equivalence methods.

The paper is structured as follows. In Section 2, we describe the DA model and we show that for binary prospects DA is a special case of rank-dependent utility. In Section 3, we describe how DA can be measured by the tradeoff method. Section 4 briefly describes how the data in Abdellaoui (2000) and Bleichrodt and Pinto (2000), that we used to elicit and test DA, were collected. Section 5 presents the results. The data suggest that DA is too parsimonious. Rather than being constant, which is what DA predicts, disappointment aversion increases with the probability of obtaining an elation outcome. In Section 6, we examine to what extent our findings are consistent with the other theories of disappointment aversion that have been proposed in the literature. We also performed a curve-fitting of a two-parameter functional form for disappointment aversion that allows 
for non-constant disappointment aversion. The results of this curve-fitting are mixed. Section 7 concludes.

\section{Disappointment aversion theory}

Let $X$ be a set of outcomes, elements of which are denoted by $x, y$. We assume that outcomes are real numbers. In the experiments reported below, outcomes designate monetary gains and losses and life-years.

Let $P$ be the set of prospects. A typical prospect is denoted $\left(p_{1}: x_{1}, \ldots, p_{n}: x_{n}\right)$, yielding outcome $x_{i}$ with probability $p_{i}, i=1, \ldots, n$, where the probabilities $p_{i}$ sum to one. A prospect is riskless if $x_{1}=\cdots=x_{n}$ or if $p_{i}=1$ for some $i$. A preference relation $\succcurlyeq$ is defined over $P$. As usual, $\succ$ denotes the asymmetric part of $\succcurlyeq$ and $\sim$ denotes its symmetric part. Preferences over outcomes are derived from preferences over riskless prospects. For convenience, we assume that $\succcurlyeq$ is monotonic: higher outcomes are preferred to lower outcomes. We denote prospects in a rank-ordered way, i.e., it is implicit in the notation $\left(p_{1}: x_{1}, \ldots, p_{n}: x_{n}\right)$ that $x_{1} \geqslant \cdots \geqslant x_{n}$.

Let $q$ be any prospect $\left(q_{1}: x_{1}, \ldots, q_{n}: x_{n}\right)$. Then there will be some $1 \leqslant k \leqslant n$ such that for all $i \leqslant k, \quad x_{i} \succcurlyeq q$ and for all $i>k, q \succcurlyeq x_{i}$. We can decompose $q$ into prospects $r=\left(r_{1}: x_{1}, \ldots, r_{k}: x_{k}\right)$ and $s=\left(s_{k+1}: x_{k+1}, \ldots, s_{n}: x_{n}\right)$ such that $q=\alpha r+(1-\alpha)$ s. Hence, all outcomes of $r$ are at least as good as $q$ and $q$ is at least as good as all outcomes of $s$. The prospect $r$ is the elation component of $q$, which is unambiguously better than $q$ itself and the prospect $s$ is the disappointment component of $q$, which is unambiguously worse than $q$ itself. The parameter $\alpha$ can be interpreted as the probability that an elation outcome obtains. Disappointment aversion theory (DA) holds if prospect $q=\left(q_{1}: x_{1}, \ldots\right.$, $\left.q_{n}: x_{n}\right)=\alpha r+(1-\alpha) s$ is evaluated by

$$
\gamma(\alpha) \sum_{i=1}^{k} r_{i} U\left(x_{i}\right)+(1-\gamma(\alpha)) \sum_{i=k+1}^{n} s_{i} U\left(x_{i}\right)
$$

and preferences and choices correspond with this evaluation. In Eq. (1), $U$ is a utility function from the set of outcomes to the reals and $\gamma$ is a unique function from $[0,1]$ to $[0,1]$ defined by $\gamma(\alpha)=\frac{\alpha}{1+(1-\alpha) \beta}$ with $\beta \in(-1, \infty)$. The parameter $\beta$ is the disappointment aversion parameter. Gul (1991) defined $\succcurlyeq$ to be disappointment averse if $\beta \geqslant 0$ and elation loving if $\beta \in(-1,0]$. Gul (1991, Theorem 5) showed that, ceteris paribus, the more risk averse a decision maker is the higher is $\beta$. Expected utility is the special case of Eq. (1) where $\beta=0$. Eq. (1) shows that $\beta$ is the only parameter that the DA model adds to expected utility. Because disappointment aversion can be characterized through one parameter, disappointment aversion, unlike risk aversion, is a global property. Gul (1991, Theorem 2) showed that in the Allais paradox $\beta>0$. In their parametric estimation of the DA functional, Hey and Orme (1994) observed both positive and negative estimates of $\beta$.

For binary prospects $(p: x ; y)$, which give $x$ with probability $p$ and $y$ with probability $1-p, x>y$, the elation component is $x$, the disappointment component is $y$, and $\alpha=p$ so that the DA model reduces to

$$
\gamma(p) U(x)+(1-\gamma(p)) U(y) .
$$

It is easy to verify that $\gamma(0)=0, \gamma(1)=1$ and that $\gamma$ is increasing in $p$. Hence, $\gamma$ satisfies the requirements for a probability weighting function in rank-dependent utility (Quiggin, 


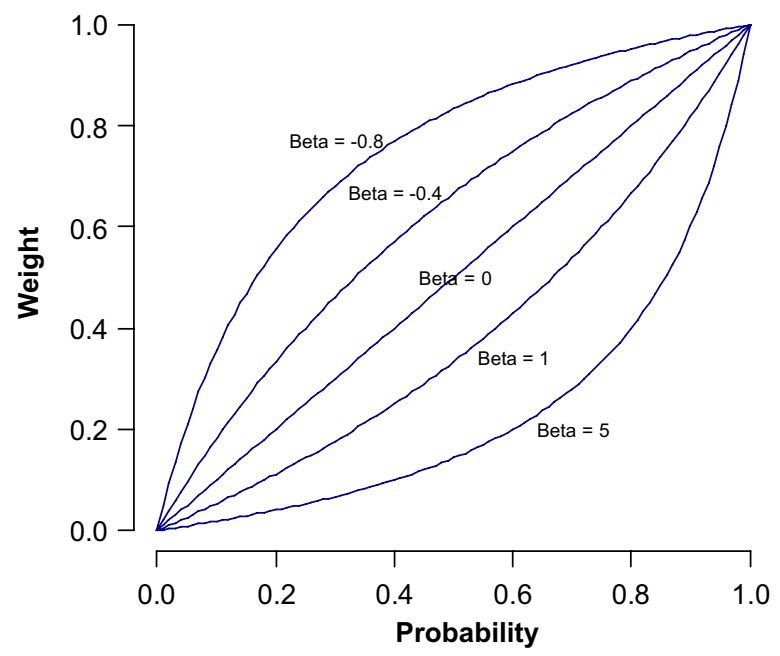

Fig. 1. Probability weighting functions implied by different values of beta.

1981) and it follows from Eq. (2) that, for binary prospects, DA reduces to a special form of rank-dependent utility.

The assumption that disappointment aversion is a global property implies restrictions on the shape of the probability weighting function when we consider DA as a special case of rank-dependent utility. Fig. 1 displays the shape of the probability weighting function for several values of $\beta$. The figure shows that in DA the probability weighting function is either concave, implying overweighting of probabilities, when the decision maker is elation loving, or convex, implying underweighting of probabilities when the decision maker is disappointment averse. DA cannot accommodate a probability weighting function that has both concave and convex parts.

Several empirical studies have shown, however, that the probability weighting function is commonly inverse S-shaped implying overweighting of small probabilities and underweighting of larger probabilities (e.g. Abdellaoui, 2000; Bleichrodt \& Pinto, 2000; Gonzalez \& Wu, 1999; Tversky \& Kahneman, 1992) although convex probability weighting has also been observed (Jullien \& Salanié, 2000; van de Kuilen, Wakker, \& Zou, 2006). The inverse $\mathrm{S}$-shape implies that people are particularly sensitive to small changes in probability from impossible to possible, a phenomenon referred to as the possibility effect, and from possible to certain, referred to as the certainty effect. The possibility effect and the certainty effect cannot be captured both by DA. DA is consistent with the possibility effect when the decision maker is elation loving and with the certainty effect when the decision maker is disappointment averse. If a decision maker behaves both according to the certainty effect and the possibility effect then we expect to find that the disappointment aversion parameter is not constant, but starts off negative and increases with the probability of the elation outcome.

\section{The tradeoff method}

The elicitation procedure for DA consists of two stages. In the first stage the utility function is elicited. Given $u(\cdot)$, the second stage elicits the disappointment aversion 
parameter $\beta$ from one single indifference. By eliciting several indifferences, we can test the stability of $\beta$ and hence the validity of the DA model. As will become apparent, our elicitation imposes no assumptions on the utility function or on $\beta$. Hence, the elicitation and test of the DA model are parameter-free.

The elicitation of $U$ and $\beta$ was performed by the tradeoff method (Wakker \& Deneffe, 1996). Wakker and Deneffe showed that the tradeoff method elicits valid utilities under rank-dependent utility. The tradeoff method only uses binary prospects and, as we showed in Section 2, for binary prospects, DA is a special case of rank-dependent utility. Therefore, the tradeoff method can also be used to elicit the utility function under DA.

\subsection{Elicitation of utility}

The first step in the tradeoff method is to fix two gauge outcomes $R$ and $r$, a starting outcome $x_{0}$ and a probability $p$. A value $x_{1}$ is elicited so that the subject is indifferent between $\left(p: x_{0} ; R\right)$ and $\left(p: x_{1} ; r\right)$. We then substitute the elicited number $x_{1}$ for $x_{0}$ and elicit the value $x_{2}$ so that the subject is indifferent between $\left(p: x_{1} ; R\right)$ and $\left(p: x_{2} ; r\right)$. By repeating this procedure, a standard sequence $x_{0}, x_{1}, \ldots, x_{k}$ results where for $j \in\{l, \ldots, k\}$ we have $\left(p: x_{j-1} ; R\right) \sim\left(p: x_{j} ; r\right)$. For monetary gains and life-years, we chose the gauge outcomes $\mathrm{R}$ and $\mathrm{r}$ so that $R>r$. It follows that the standard sequence is increasing, i.e., $x_{0}<\ldots<x_{k}$. For monetary losses, we chose $r>R$ and the standard sequence is decreasing, i.e., $x_{0}>x_{1}>\cdots>x_{k}$.

Evaluating the indifferences $\left(p: x_{j-1} ; R\right) \sim\left(p: x_{j} ; r\right)$ gives by DA

$$
\gamma(p) U\left(x_{j-1}\right)+(1-\gamma(p)) U(R)=\gamma(p) U\left(x_{j}\right)+(l-\gamma(p)) U(r)
$$

or

$$
U\left(x_{j}\right)-U\left(x_{j-1}\right)=\frac{1-\gamma(p)}{\gamma(p)}(U(R)-U(r)) .
$$

Since this holds for all $j$ in $\{1, \ldots, k\}$, the utility difference between two successive elements of the standard sequence is constant.

For monetary gains and life-years, we normalized utility so that $U\left(x_{0}\right)=0$ and $U\left(x_{k}\right)=1$. It follows that $U\left(x_{j}\right)=j / k, j=0, \ldots, k$. For monetary losses, we normalized utility so that $U\left(x_{0}\right)=0$ and $U\left(x_{k}\right)=-1$ and, therefore, $U\left(x_{j}\right)=-j / k, j=0, \ldots, k$.

\subsection{Elicitation of disappointment aversion}

After the elicitation of the utility function through the construction of a standard sequence $x_{0}, x_{1}, \ldots, x_{k}$, the only remaining parameter that needs to be assessed is the disappointment aversion parameter $\beta$. Suppose that the standard sequence is increasing. The most straightforward method to elicit $\beta$ is to fix an element $x_{j}$ of the standard sequence and then to elicit the probability $p j$ that makes the subject indifferent between $x_{j}$ for sure and the prospect $\left(p_{j}: x_{k} ; x_{0}\right)$. It follows from DA that

$$
U\left(x_{j}\right)=\gamma\left(p_{j}\right) U\left(x_{k}\right)+\left(1-\gamma\left(p_{j}\right)\right) U\left(x_{0}\right),
$$

or $\gamma\left(p_{j}\right)=\frac{U\left(x_{j}\right)-U\left(x_{0}\right)}{U\left(x_{k}\right)-U\left(x_{0}\right)}=j / k$. When the standard sequence is decreasing, then the probability $p_{j}$ is elicited that makes the subject indifferent between and $\left(p_{j}: x_{0} ; x_{k}\right)$. This $k-j$ indifference implies by DA that $\gamma\left(p_{j}\right)=\frac{k-j}{k}$. 
The resulting disappointment aversion parameter is given by

$$
\beta=\frac{p_{j}-\gamma\left(p_{i}\right)}{\gamma\left(p_{j}\right)\left(1-p_{j}\right)} .
$$

By varying $x_{j}$ we can elicit different observations of $\beta$. As noted in Section 2, DA predicts that the elicited values of $\beta$ are all equal; any differences must due to random error.

A disadvantage of the above procedure may be that the question format and the response scale differ between the first stage, the elicitation of the utility function, and the second stage, the elicitation of the disappointment aversion parameter. In the first stage, both prospects are risky and the response scale is outcome $\left(x_{j}\right)$, in the second stage one of the prospects is riskless and the response scale is probability $\left(p_{j}\right)$. Several studies suggest that varying the response scale may lead to distortions in the elicited preferences (Delquié, 1993; Tversky, Sattath, \& Slovic, 1988). One way to mitigate the effect of such distortions is to obfuscate that probability is the response scale, for example by eliciting indifference through a sequence of choices.

An alternative procedure to elicit the disappointment aversion parameter, which holds the question format and the response scale fixed across the two stages and, therefore, may be less susceptible to distortions, is the following. Fix three different elements of the standard sequence that was elicited in the first stage, say $x_{j 1}, x_{j 2}, x_{j 3}$ with $x j_{1}<x_{j 2}<x_{j 3}$. Also fix a probability $p$. Then elicit the outcome $z$ so that the subject is indifferent between $\left(p: x_{j 3} ; x_{j 2}\right)$ and $\left(p: z ; x_{j 3}\right)$. It follows from DA that

$$
\gamma(p)=\frac{\frac{j_{2}}{k}-\frac{j_{1}}{k}}{\left(U(z)-\frac{j_{3}}{k}\right)\left(\frac{j_{2}}{k}-\frac{j_{1}}{k}\right)}
$$

and the formula for $\beta$ follows from Eq. (6). A test of DA is obtained by varying the probability $p$ across questions, thereby eliciting several values of $\beta$. Again, DA predicts that the obtained values are all equal, except for random error.

A disadvantage of the second elicitation procedure is that $U(z)$ is in general unknown and has to be determined through interpolation. As long as successive elements of the standard sequence are relatively close this will cause no problems.

A potential problem of both approaches is error propagation: an error made during the elicitation of the utility function affects the elicitation of subsequent elements of the standard sequence and of the disappointment aversion parameter. Abdellaoui (2000) and Bleichrodt and Pinto (2000) showed that error propagation caused no problems for the elicitation of the probability weighting function, and thus of $\beta$, by the two methods described above (see also Abdellaoui, Vossmann, \& Weber, 2005).

\subsection{Use of our method in applications}

The above procedure requires that outcomes are quantitative. For qualitative outcomes like health states the tradeoff method cannot be applied. The way to proceed for such outcomes is as follows. First apply our method for quantitative outcomes to determine $\beta$. For example, in the health domain one could determine $\beta$ by using life durations as outcomes. Knowledge of $\beta$ can then be used to correct traditional utility measurements for disappointment. Consider, for example, the probability equivalence method, which is widely used in medical decision making to determine the utility of health states. A common 
question in medical decision making is to ask a patient to specify the probability $p$ that makes him indifferent between a given impaired health state, say back pain, for sure and a treatment option that gives probability $p$ of full health and probability $1-p$ of death within a week. Under expected utility and the scaling $U$ (full health) $=1$ and $U$ (death within a week $)=0$ it follows that the utility of back pain is equal to $p$. The same scaling implies that under the DA model the utility of back pain is equal to $\gamma(p)$. Knowledge of $\beta$ determines $\gamma(p)$ and allows to reassess existing utility measurements and to correct these for disappointment so as to arrive at more realistic utilities for health.

\section{Experiments}

\subsection{First experiment: monetary gains and losses}

The first elicitation/test of the DA model that we performed used the data from Abdellaoui (2000). In this study, monetary outcomes were used. Forty French undergraduate and Ph.D. students in economics participated, who were paid FF 150 (approximately €23) for their participation. Separate elicitations of $U$ and $\beta$ were performed for gains and for losses. Subjects were told that for gains one subject would be randomly selected to play for real money one of his answers in the first stage of the elicitation and one of his answers in the second stage of the elicitation. For losses a similar incentive mechanism is ethically objectionable and, hence, no real incentives were used for losses.

To elicit the utility function, the outcomes $\left|x_{0}\right|,|R|$, and $|r|$ were fixed at FF $1000(€ 150)$, FF 500 (€75), and 0 , respectively. The probability $p$ was set equal to $2 / 3$. Standard sequences of seven elements, $x_{0}, x_{1}, \ldots, X_{6}$, were constructed. Hence, subjects faced the options $\left(2 / 3:-500 ; x_{j}\right)$ and $\left(2 / 3: x_{j+1} ; 0\right)$ for gains and the options $\left(1 / 3:-500 ; x_{j}\right)$ and $\left(1 / 3: 0 ; x_{j+1}\right)$ for losses, $j=0, \ldots, 5$. As has been mentioned before, the standard sequences were increasing for gains and decreasing for losses. Indifferences between the options were elicited by a choice-based procedure.

To elicit $\beta$, the first procedure described in Section 3.2 was used, where, to avoid distortions, the indifference probabilities were elicited by a choice-based procedure. Each subject was asked a series of choices aiming to determine the probabilities $p 1, \ldots, p 5$ that made him indifferent between $x_{j}$ and $\left(p_{j}: x_{6} ; x_{0}\right)$ for gains and between $x_{j}$ and $\left(p_{j}: x_{0} ; x_{6}\right)$ for losses, $j=1, \ldots, 5$. Several consistency questions were included, which indicated no systematic inconsistencies. A more detailed description of the experimental procedure is given in Abdellaoui (2000).

\subsection{Second experiment: life-years}

The second elicitation/test of the DA model used the data from Bleichrodt and Pinto (2000). The outcomes in this study were life-years. Fifty-one Spanish undergraduate economics students participated, who were paid Ptas 5000 (approximately €30). Obviously, real incentives could not be used.

Prospects were described as medical treatments. Subjects were asked to imagine that they displayed symptoms that implied that they had one of two diseases; it was unknown from which disease they suffered. Subjects were asked to choose between two treatments to cure the symptoms. 
To elicit the utility function, the outcomes $x_{0}, R$, and $r$ were fixed at 0 years, 55 years, and 45 years respectively. Hence, in the second experiment the gauge outcomes were more attractive than the elements of the standard sequence. This does not affect the conclusions of Section 3.1 as long as $r$ is at least as large as the final element of the standard sequence. The probability $p$ was set equal to $1 / 2$. Standard sequences of seven elements, $x_{0}, x_{1}, \ldots, x_{6}$, were constructed. Hence, subjects faced the options $\left(1 / 2: 55\right.$ years; $\left.x_{j}\right)$ and $\left(1 / 2: 45\right.$ years; $\left.x_{j}+1\right), j=0, \ldots, 5$. Indifferences were elicited by matching.

To elicit $\beta$, the second procedure described in Section 3.2. was used. The utility of $z$ was estimated both by linear interpolation and by interpolation based on a power function estimated from the data of the first stage (the utility elicitation). Five probabilities $p_{i}$ were used: $0.10,0.25,0.50,0.75$, and 0.90 . For probabilities $0.10,0.25$, and 0.50 , subjects were asked to state their indifference value $\mathrm{z}$ in the comparison between $\left(p: x_{4} ; x_{3}\right)$ and $\left(p: x_{5} ; z\right){ }^{1}$ For probabilities 0.75 and 0.90 , subjects were asked to state their indifference value $z$ in the comparison between $\left(p: x_{3} ; x_{2}\right)$ and $\left(p: z ; x_{1}\right)$. The value of $\beta$ then followed from Eqs. (6) and (7). Several consistency questions were included. They indicated no systematic inconsistencies. A more detailed description of the experimental procedure is given in Bleichrodt and Pinto (2000).

\section{Results}

All responses were included in the analysis of the first experiment. In the analysis of the second experiment, the responses of two subjects were discarded. One subject refused to make any tradeoffs, for the other subject $x_{6}>45$ years $=r$ and hence rank-ordering of prospects was violated.

\subsection{Utility}

Fig. 2 displays the elicited utility functions across the three outcome domains. The figure shows that the utility function is mildly concave for gains, mildly convex for losses, and concave for life-years. To smoothen out response errors, for each subject a parametric estimation of the utility function was performed where it was assumed that the utility function was a power function. The power function was chosen because previous studies observed that it provided an excellent fit to the data. Median power coefficients were 0.89 for gains, 0.92 for losses and 0.77 for life-years.

The aggregate data were confirmed by individual-subject analysis. Both for monetary gains and for life-years, most subjects had a concave utility function. For both outcome domains, there were significantly more subjects with concave than subjects with convex utility functions $(p<0.01$, binomial, one-tailed). For monetary losses, the modal shape of the utility function was convex. There were significantly more convex utility functions than concave utility functions ( $p=0.025$, binomial, one-tailed). Further details, e.g. with respect to the classification used, are in Abdellaoui (2000) and in Bleichrodt and Pinto (2000).

\footnotetext{
${ }^{1}$ Then $\gamma(p)=\frac{(1 / 2)-U(z)}{(2 / 3)-u(z)}$.
} 

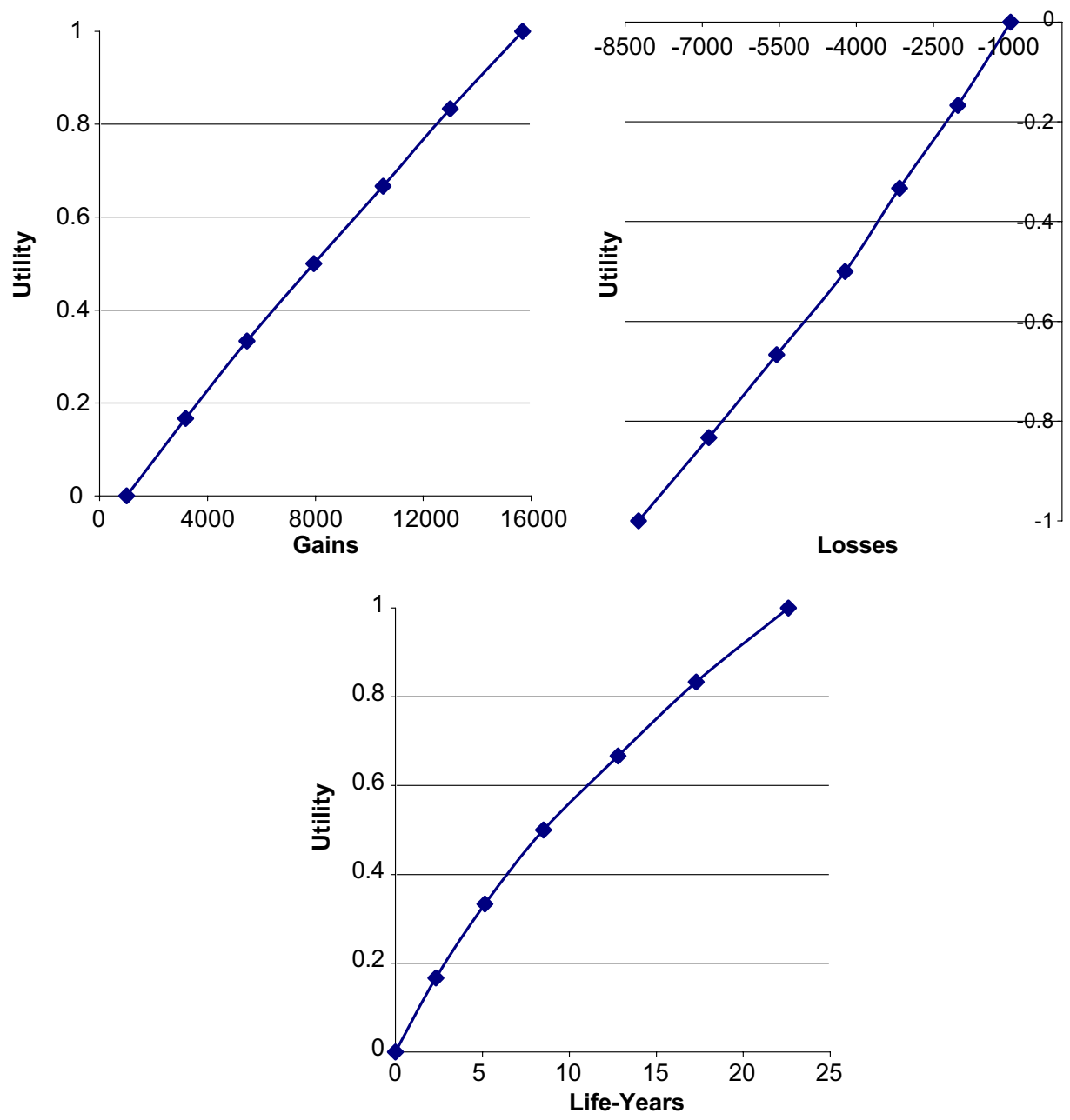

Fig. 2. Elicited utility functions for gains, losses, and life-years.

\subsection{Disappointment aversion}

Table 1 gives an overview of the individual results. The table shows that for each of the three outcome domains the proportion of disappointment averse subjects, i.e. those for whom $\beta>0$, increased with the probability of the elation outcome. That disappointment aversion increases with the probability of the elation outcome seems intuitive. If the probability of success is small, people will not really count on it and will feel little disappointment when the elation outcome does not obtain. If the probability of success is high, people will feel more disappointed when the elation outcome does not obtain.

Fig. 3 plots the probabilities and the median values for the disappointment aversion parameter $\beta$ for gains, losses, and life-years. For life-years only the relationship under linear interpolation is displayed. The relationship under power interpolation is similar. 
Table 1

Proportion of disappointment averse subjects

\begin{tabular}{llcc}
\hline Elicitation & Gains $(\%)$ & Losses $(\%)$ & Life-years (\%) \\
\hline 1 & 37.5 & 5 & 4.2 \\
2 & 57.5 & 22.5 & 25.6 \\
3 & 75 & 40 & 74.5 \\
4 & 90 & 52.5 & 83.7 \\
5 & 97.5 & 72.5 & 91.3 \\
\hline
\end{tabular}

Note: For gains and losses elicitation $j$ measured $p_{j}=w^{-1}(j / 6), j=1, \ldots, 5$. For life-years elicitation $j$ measured $w\left(p_{j}\right)$ with $p_{1}, \ldots, p_{5}=0.10,0.25,0.50,0.75,0.90$.

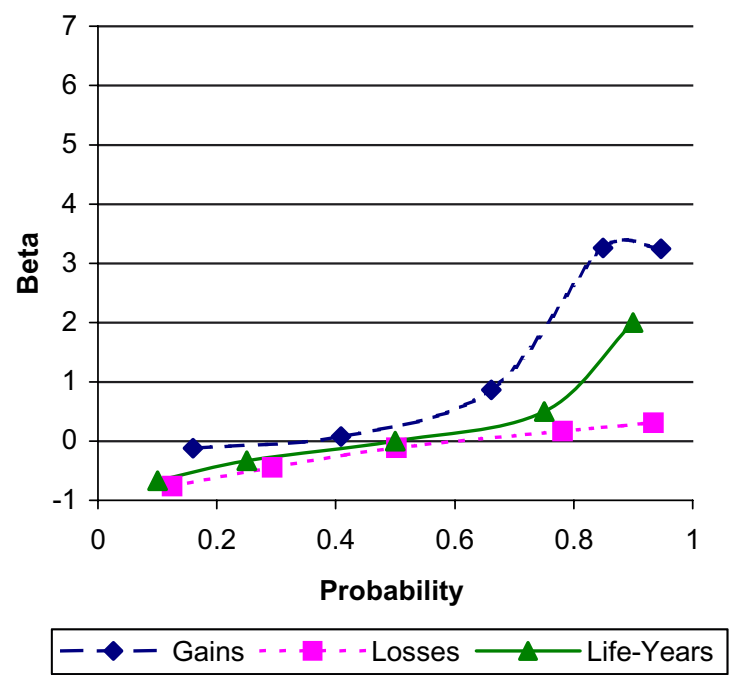

Fig. 3. Elicited values of the disappointment aversion parameter.

Fig. 3 confirms the individual data and shows that $\beta$ is not constant at the aggregate level either. The hypothesis of constant $\beta$ is rejected for all outcome domains $(p<0.001$ both by ANOVA with repeated measures and by the nonparametric Friedman test). Since expected utility is the special case of DA where $\beta=0$, the data also reject expected utility.

In terms of the probability weighting function, the finding that disappointment aversion increases with the probability of the elation outcome entails that the data are consistent with a probability weighting function that overweights small probabilities and underweights large probabilities, and thus with the joint existence of the possibility effect and the certainty effect.

Table 1 and Fig. 3 suggest that, for the probabilities considered, disappointment aversion is higher for gains than for losses. Sign-dependence of the disappointment aversion parameter is confirmed by a signed rank-test $(z=-3.42, p=0.001)$. The median disappointment aversion parameter also differs significantly between gains and life-years $(z=-4.32, p<0.001$, Mann-Whitney Test). No significant differences were found between losses and life-years $(z=-1.485, p=0.138$, Mann-Whitney Test). In comparing 
the results for money amounts and for life-years, one should keep in mind that the procedure for eliciting $\beta$ was different for money than for life duration. This difference in elicitation procedure may have confounded the comparison. We return to this issue in Section 7.

\section{Other disappointment models}

Let us now briefly consider the question whether the other disappointment models that have been proposed in the literature can account for our finding that disappointment aversion, and, hence, risk aversion, increases with the probability of the elation component. A caveat that should be kept in mind in performing such an analysis is that our method was not specifically designed to elicit these other disappointment theories.

Bell (1985) proposed the following functional form for a binary prospect $(p: x ; y), x>y$,

$$
p x+(1-p) y+(e-d) p(1-p)(x-y),
$$

where $e$ is a constant reflecting the degree to which a unit of elation affects the decision maker and $d$ is a constant that reflects the degree to which a unit of disappointment affects a decision maker. Eq. (8) can be rewritten as $w(p) x+(1-w(p)) y$ with

$$
w(p)=(p+(e-d) p(1-p)) .
$$

Bell's model is a special form of rank-dependent utility with linear utility and the probability weighting function given by Eq. (9). It is obvious from this equation that $w(p)$ either underweights or overweights probabilities but cannot account for the inverse S-shape. Consequently, our data are not consistent with Bell's model of disappointment aversion and neither with the model of Jia et al. (2001), which is identical to Bell's model for two outcome prospects. The model of Grant and Kajii (1998) corresponds to rank-dependent utility with a power probability function $w(p)=p^{\alpha}$ and can only account for underweighting of probabilities, i.e. disappointment aversion. Therefore, their model is not consistent with our data either.

Delquié and Cillo (2006a) suggested that $(p: x ; y)$ be evaluated as

$$
p u(x)+p(1-p) \mathscr{E}(u(x)-u(y))+(1-p) u(y)-(1-p) p \mathscr{D}(u(x)-u(y)),
$$

where $\mathscr{E}$ is a real-valued function capturing sensitivity to elation and $\mathscr{D}$ is a real-valued function capturing sensitivity to disappointment. Both $\mathscr{E}$ and $\mathscr{D}$ are nondecreasing and satisfy $\mathscr{E}(0)=\mathscr{D}(0)=0$. Delquié and Cillo's model cannot account for the increasing relationship between disappointment aversion and the probability of the elation component: disappointment aversion is the same when the probability of the elation component is, say, 0.10 as when it is 0.90 . Hence, Delquié and Cillo's model cannot account for our data.

Finally, Loomes and Sugden (1986) proposed the following functional form for the evaluation of $(p: x ; y)$ :

$$
\begin{aligned}
p u(x) & +p \mathscr{D}(u(x)-(p u(x)+(1-p) u(y)))+(1-p) u(y)+(1-p) D(u(y)-(p u(x) \\
& +(1-p) u(y))) \\
= & p u(x)+p \mathscr{D}((1-p)(u(x)-u(y)))+(1-p) u(y)-(1-p) \mathscr{D}(p(u(x)-u(y))) .
\end{aligned}
$$


Table 2

Parameter estimates for the exponential form $\beta=a+e^{b p}$

\begin{tabular}{lll}
\hline & $a$ & $b$ \\
\hline Gains & -1.06 & 1.62 \\
& $(-1.97,0.62)$ & $(0.80,2.74)$ \\
Losses & -1.70 & 0.50 \\
& $(-1.99,-1.40)$ & $(0.05,1.82)$ \\
Life-years & -1.79 & 1.33 \\
& $(-1.99,-1.60)$ & $(0.67,1.77)$ \\
\hline
\end{tabular}

Interquartile ranges of individual parameters of $a$ and $b$ are in parentheses.

In Loomes and Sugden's model the prior expectation of a prospect is its expected utility. The function $\mathscr{D}$ captures sensitivity to elation when the utility of an outcome is larger than the prior expectation and sensitivity to disappointment when the utility of an outcome is less than the prior expectation. $\mathscr{D}$ is nondecreasing, satisfies $\mathscr{D}(0)=0$ and $\mathscr{D}(-x)=-\mathscr{D}(x)$ for all $x>0$, and is convex on its positive domain and concave on its negative domain. Under these assumptions Loomes and Sugden's (1986) model predicts that disappointment aversion increases with the probability of the elation component $\mathrm{x}$, a prediction that is in accord with our data.

Loomes and Sugden's (1986) model is more sophisticated, however, than the DA model and there exists as yet no method to elicit their model. We therefore explored whether adding one additional parameter to the DA model, which reflects how disappointment aversion varies with the probability of obtaining an elation outcome, could account for our data. This extended DA model is still tractable and may suggest ways to generalize the DA model. Of the two-parameter functional forms that we examined (linear, quadratic, power, exponential, expo-power (Saha, 1993), and HARA (Merton, 1971)) the exponential form $\beta=a+e^{b \alpha}$, where $\alpha$ is the probability of obtaining an elation outcome, fitted the data best based on the criterion "minimize the sum of the squared errors subject to $\beta>-1 . "$

The curve-fitting yielded mixed results. As Table 2 shows, the median estimates for $b$ differed substantially across the three domains. The difference was significant between losses and gains $(p=0.012)$; the other differences were not significant. There was a lot of variation in the elicited values of $b$ at the individual level. The fit was good for losses and for life-years, but less so for gains.

\section{Conclusion}

This paper has proposed a method to measure DA, a popular nonexpected utility model that generalizes expected utility by adding one parameter. The availability of an elicitation method is important both for descriptive purposes, to test the descriptive accuracy of the DA model, and for applications as our method allows separating utility and disappointment. Moreover, knowledge of the disappointment parameter $\beta$ makes it possible to correct utility measurements using traditional measurement tools, such as the probability equivalence and certainty equivalence methods, for disappointment.

Our descriptive test of the DA model showed that disappointment aversion is not constant, as DA assumes, but varies with the probability of the elation outcome. This pattern appears robust: we found it both for monetary and for health outcomes, both for gains and 
for losses, and both for hypothetical and for incentivized questions. We found evidence of sign-dependence of disappointment aversion: disappointment aversion was higher for gains than for losses. Evidence on outcome-dependence was less clear. The conclusions about outcome-dependence are necessarily tentative as the elicitation processes differed for money outcomes and for life duration. The extent to which these differences confound the comparison between the two domains is unclear. It is well known that preference measurements are generally volatile and depend on the way preferences are measured. On the other hand the different elicitation procedures need not lead to systematic biases in the elicited values of the disappointment parameter. Consider for example scale compatibility, which says that the decision maker will overweight the response scale used. If probability is used as the response scale, scale compatibility predicts that subjects will focus more on the probabilities in responding to the questions than when outcome is used as the response scale. Scale compatibility does not predict on which probability subjects will focus. If they focus on the probability of the elation outcome then the disappointment parameter will be lower than when they focus on the probability of the disappointment outcome. The potential bias arising from the difference in elicitation procedure is ambiguous.

Of the other disappointment models that have been proposed in the literature, our data are only consistent with the model of Loomes and Sugden (1986). Our method for measuring the DA model does not apply to Loomes and Sugden's model, however, and restraint should be exercised in drawing strong inferences about the validity of the other disappointment models based on our data alone. Future research may try to construct a method that allows measuring Loomes and Sugden's model to obtain a proper test of that theory and to improve its applicability.

Another possibility would be to generalize the DA model by allowing disappointment aversion to vary with the probability of the elation outcome, while keeping the model tractable. We performed some preliminary analysis on this topic by fitting two-parameter functional forms to our data. The findings of our curve-fitting exercise are mixed: they are more satisfactory for losses and life-years than for gains. An interesting test that may be addressed in future research is whether the fitted curves are consistent with other available data and how well they predict choices between prospects involving more than two outcomes.

\section{Acknowledgements}

Peter Wakker and two anonymous reviewers gave many helpful comments. Mohammed Abdellaoui's research was supported by CNRS, France. Han Bleichrodt's research was made possible by Grants from the Royal Netherlands Academy of Science and from the Netherlands Organisation for Scientific Research (NWO).

\section{References}

Abdellaoui, M. (2000). Parameter-free elicitation of utility and probability weighting functions. Management Science, 46, 1497-1512.

Abdellaoui, M., Vossmann, F., \& Weber, M. (2005). Choice-based elicitation and decomposition of decision weights for gains and losses under uncertainty. Management Science, 51, 1384-1399.

Bell, D. E. (1985). Disappointment in decision making under uncertainty. Operations Research, 33, 1-27.

Blavatskyy, P. R. (2006). Violations of betweenness or random errors? Economics Letters, 91, 34-38.

Bleichrodt, H., \& Pinto, J. L. (2000). A parameter-free elicitation of the probability weighting function in medical decision analysis. Management Science, 46, 1485-1496. 
Camerer, C. F., \& Ho, T.-H. (1994). Nonlinear weighting of probabilities and violations of the betweenness axiom. Journal of Risk and Uncertainty, 8, 167-196.

Chew, S. H., \& Waller, W. (1986). Empirical tests of weighted utility theory. Journal of Mathematical Psychology, $30,55-62$.

Conlisk, J. (1989). Three variants on the Allais paradox. American Economic Review, 79, 392-407.

Delquié, P. (1993). Inconsistent tradeoffs between attributes: New evidence in preference assessment biases. Management Science, 39, 1382-1395.

Delquié, P., \& Cillo, A. (2006a). Disappointment without prior expectation: A unifying perspective on decision under risk. Journal of Risk and Uncertainty, 33, 197-215.

Delquié, P., \& Cillo, A. (2006b). Expectations, disappointment, and rank-dependentprobability weighting. Theory and Decision, 60, 193-206.

Farquhar, P. (1984). Utility assessment methods. Management Science, 30, 1283-1300.

Gonzalez, R., \& Wu, G. (1999). On the form of the probability weighting function. Cognitive Psychology, 38, $129-166$.

Grant, S., \& Kajii, A. (1998). Ausi expected utility: An anticipated utility theory of relative disappointment aversion. Journal of Economic Behavior and Organization, 37, 277-290.

Gul, F. (1991). A theory of disappointment aversion. Econometrica, 59, 667-686.

Harless, D., \& Camerer, C. F. (1994). The predictive utility of generalized expected utility theories. Econometrica, $62,1251-1289$.

Hey, J. D., \& Orme, C. (1994). Investigating generalizations of expected utility theory using experimental data. Econometrica, 62, 1291-1326.

Jia, J., Dyer, J. S., \& Butler, J. C. (2001). Generalized disappointment models. Journal of Risk and Uncertainty, $22,59-78$.

Jullien, B., \& Salanié, B. (2000). Estimating preferences under risk: The case of racetrack bettors. Journal of Political Economy, 108, 503-530.

Kahneman, D., \& Tversky, A. (1979). Prospect theory: An analysis of decision under risk. Econometrica, 47, 263-291.

Loomes, G., \& Segal, U. (1994). Observing different orders of risk aversion. Journal of Risk and Uncertainty, 9 , $239-256$.

Loomes, G., \& Sugden, R. (1986). Disappointment and dynamic consistency in choice under uncertainty. Review of Economic Studies, 53, 271-282.

McCord, M., \& de Neufville, R. (1986). Lottery equivalents: Reduction of the certainty effect problem in utility assessment. Management Science, 32, 56-60.

Merton, R. C. (1971). Optimum consumption and portfolio rules in a continuous-time model. Journal of Economic Theory, 3, 373-413.

Morone, A., \& Schmidt, U. (2002). An experimental investigation of alternatives to expected utility using pricing data. Working Paper, University of Hannover.

Neilson, W. S. (1992). A mixed fan hypothesis and its implications for behavior towards risk. Journal of Economic Behavior and Organization, 19, 197-212.

Quiggin, J. (1981). Risk perception and risk aversion among Australian farmers. Australian Journal of Agricultural Economics, 25, 160-169.

Saha, A. (1993). Expo-power utility: A 'flexible' form for absolute and relative risk aversion. American Journal of Agricultural Economics, 75, 905-913.

Savage, L. J. (1954). The foundations of statistics. New York: Wiley.

Starmer, C. (2000). Developments in non-expected utility theory: The hunt for a descriptive theory of choice under risk. Journal of Economic Literature, 28, 332-382.

Tversky, A., \& Kahneman, D. (1992). Advances in prospect theory: Cumulative representation of uncertainty. Journal of Risk and Uncertainty, 5, 297-323.

Tversky, A., Sattath, S., \& Slovic, P. (1988). Contingent weighting in judgment and choice. Psychological Review, 95, 371-384.

van de Kuilen, G., Wakker, P. P., \& Zou, L. (2006). A midpoint technique for easily measuring prospect theory's probability weighting. Working Paper, University of Amsterdam and Erasmus University Rotterdam.

Wakker, P. P., \& Deneffe, D. (1996). Eliciting von Neumann-Morgenstern utilities when probabilities are distorted or unknown. Management Science, 42, 1131-1150.

Yaari, M. E. (1987). The dual theory of choice under risk. Econometrica, 55, 95-115. 